\title{
Error analysis for a free-hand three-dimensional ultrasound system for neuronavigation
}

\author{
Alexander Hartov, Ph.D., Symma D. Eisner, M.S., David, W. Roberts, M.D., Keith D. Paulsen, \\ Ph.D., Leah A. Platenik, B.S., and Michael I. Miga, Ph.D.
}

Thayer School of Engineering, Dartmouth College, and Dartmouth Medical School, Lebanon, New Hampshire

Image-guided neurosurgery that is directed by a preoperative imaging study, such as magnetic resonance (MR) imaging or computerized tomography (CT) scanning, can be very accurate provided no significant changes occur during surgery. A variety of factors known to affect brain tissue movement are not reflected in the preoperative images used for guidance. To update the information on which neuronavigation is based, the authors propose the use of three-dimensional (3-D) ultrasound images in conjunction with a finite-element computational model of the deformation of the brain. The 3-D ultrasound system will provide real-time information on the displacement of deep structures to guide the mathematical model. This paper has two goals: first, to present an outline of steps necessary to compute the location of a feature appearing in an ultrasound image in an arbitrary coordinate system; and second, to present an extensive evaluation of this system's accuracy. The authors have found that by using a stylus rigidly coupled to the 3-D tracker's sensor, they were able to locate a point with an overall error of $1.36 \pm 1.67 \mathrm{~mm}$ (based on 39 points). When coupling the tracker to an ultrasound scanhead, they found that they could locate features appearing on ultrasound images with an error of $2.96 \pm 1.85 \mathrm{~mm}$ (total 58 features). They also found that when registering a skull phantom to coordinates that were defined by MR imaging or CT scanning, they could do so with an error of $0.86 \pm 0.61 \mathrm{~mm}$ (based on 20 coordinates). Based on their previous finding of brain shifts on the order of $1 \mathrm{~cm}$ during surgery, the accuracy of their system warrants its use in updating neuronavigation imaging data.

Key Words * stereotactic neurosurgery * image-guided surgery * three-dimensional ultrasound * frameless stereotaxy * three-dimensional tracking

Frameless stereotactic neurosurgery requires a preoperative imaging study in which diagnostic tools such as computerized tomography (CT) scanning or magnetic resonance (MR) imaging are performed to create a three-dimensional (3-D) anatomical map on which neuronavigation is based.[11,13] Excellent results have been obtained in locating and targeting desired anatomical features by using this technique, provided that no significant changes in brain morphology occur during surgery.[11] However, loss of accuracy results if change does occur, due to deformation caused either by the surgical procedure itself or 
simply by gravity acting on the brain in the open cranium. [5-7,12] Some investigators have attempted to remedy this problem by using intraoperative MR imaging,[14] but this comes at considerable cost. Another correctional approach relies on the use of a finite-element mechanical model of the brain that is used to predict how the brain will deform under surgical loads.[9] This model uses a 3-D representation of the brain based on the preoperative imaging study that has been processed into a mesh through segmentation and that depends on up-to-date information such as pressure or displacement at various points for its accuracy. We propose to provide timely information on the displacement of subcortical or deep structures by performing 3-D intraoperative ultrasound.

The problem of tracking ultrasound images in a 3-D representation has been of interest for several groups and has elicited two classes of solutions: tracking the imaging scanhead with an articulated arm[3] or with a tethered tracker.[4,10] The articulated-arm approach is acceptable in a limited context. In neurosurgery, for example, because the range of motion of the scanhead is limited, the bulk of the apparatus makes it a less than ideal solution in an already crowded operating field. The tethered tracker, typically a magnetic sensor, is less limited in its range of motion and very compact, consisting of a sensor approximately 1 cubic inch with a cable that extended out to the monitoring equipment (smaller sensors are available). The compromise lies in the better accuracy inherent in an articulated arm compared with the less cumbersome nature of a free-moving and unobtrusive tracker. Birkfellner, et al.,[2] have presented a detailed analysis of the accuracy one can expect from using magnetic trackers. They reported an overall accuracy in a realistic operating room (OR) environment of $6.4 \pm 2.5 \mathrm{~mm}$ in distance and $4.9 \pm$ $2^{\circ}$ in rotation for the type of apparatus we are using. We found that our apparatus performed generally better in the lab and in the OR. Leotta and colleagues[8] have investigated how to improve the accuracy of such systems as well. We have performed a number of tests to evaluate the possible use of such a tethered 3-D tracker in updating imaging data intraoperatively for the purpose of improving neuronavigation during surgery.

\section{MATERIALS AND METHODS}

\section{System Description}

In our 3-D ultrasound system the imaging scanhead is used in 3-D space that records its location, and orientation each time an image is acquired. Given an image, its location, and its orientation in space, it is possible to assign a 3-D coordinate to each pixel. Given a series of images encompassing a given volume, it is possible to reconstruct a 3-D graphic representation of that volume, provided that a sufficient number of pixels (now voxels) is available. For this purpose, we have built a system that includes an intraoperative ultrasonography station (Aloka Model 633; Corometrix Medical Systems, Wallingford, CT), a computer equipped with a frame grabber (model DT3155; Data Translation, Marlboro, MA), and a 3-D electromagnetic tracker (Flock of Birds [FOB]); Ascension Technology, Milton, VT). Custom-designed computerized software controls the image acquisition and the 3-D tracker and provides the ability to acquire images either individually or in sequences. Images can be obtained by having the surgeon or an operator press a remote button, which keeps interactions with the computer to a minimum.

\section{Stylus Calibration}

The FOB 3-D tracking system consists of a transmitter whose position is fixed and a receiver that can be moved freely and whose position is tracked in the coordinate system defined by the transmitter. In addition to reporting the receiver's position, the FOB system also reports its orientation, thus providing 
six degrees of freedom. The transmitter and receiver define two distinct coordinate systems that are related by a transformation. The FOB supplies the user with a rotation matrix, $\mathrm{M}_{3} \mathrm{X}_{3}$, and a vector, $\operatorname{Pr}_{3 \mathrm{X}}$ , indicating the position of the receiver. Thus, a point in receiver coordinates, $\mathrm{Vr}_{3 \times 1}$, can be transformed into a point in transmitter coordinates $\mathrm{Vt}_{3 \times 1}$ by the equation: (Equation 1)

$$
5 \times r_{3} \times 1=B_{3} \times 3 \times 5 \times 1+F_{3} \times 1
$$

A more convenient notation used in the context of computer graphics uses affine $\left(4 \mathrm{X}_{4}\right)$ matrices to describe transformations between coordinate systems. In this system, a point is described by a four-tuple $[\mathrm{x} \mathrm{y} \mathrm{z} 1]^{\mathrm{T}}$, and transformation matrices are used to perform both translation and rotation in a single operation. Equation 1 can be rewritten as: (Equation 2)

$$
r_{4} \times 1=T_{4} \times 4 \times S_{4} \times 1
$$

where ${ }^{t} \operatorname{Tr}_{4 \times 4}$ is the transformation matrix that relates the receiver and transmitter spaces. Its elements can be obtained from $\mathrm{M}_{3 \times 3}$ and $\operatorname{Pr}_{3 \times 1}$ : (Equation 3)

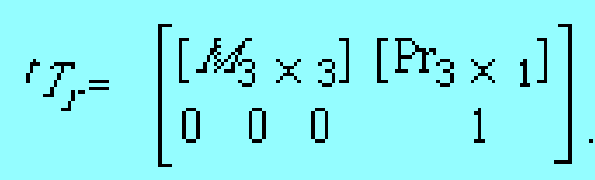

We use both systems of notation depending herein as the need arises.

In the process of registering the patient with the CT- or MR imaging-defined coordinate system, we need to identify the coordinates of arbitrary reference points in the patient. For this purpose, we use a stylus that is rigidly coupled to the receiver. To ascertain the position of the stylus tip in receiver coordinates, we use a simple calibration procedure that consists of holding the tip position fixed and recording the receiver parameters for several different positions of the stylus/receiver assembly, typically 10 times. To compute the location of the stylus tip in receiver coordinates (Vs), we use the following approach, which is outlined in the FOB manual (Ascension Technology Corp.). Given $\mathrm{n}$ positions, we have $\mathrm{n}$ equations of the form: (Equation 4)

$$
\Gamma \cdot r=B_{j} \times \quad r i r+\operatorname{Pr}_{i} \quad(i=1 \text { to } n)
$$

where $\mathrm{Vt}$ is the location of the fixed point in transmitter space, Mi the rotation matrices corresponding to each position, Vs the tip location in receiver space, which is also fixed and which we want to determine, and $\operatorname{Pr}_{i}$ the receiver positions. The location of the stylus tip, with respect to the receiver reference frame, represents only three parameters or degrees of freedom. By subtracting (n-1) equations from the first and knowing that the Vt vectors are all the same, we can write: (Equation 5)

$$
0=\left(A_{1}-A_{1}\right) \times 5+\left(\operatorname{Pr}_{1} 2 \operatorname{Pr}_{\mathrm{i}}\right) \quad(\mathrm{i}=2 \text { to } \mathrm{n})
$$

By adding the (n-1) resulting equations we obtain: (Equation 6) 


$$
\begin{aligned}
& \left(\sum_{i=2}^{n} \operatorname{Pr}_{1}-(B z-1) \cdot \operatorname{Pr} 1\right)=\left((n-1) \cdot M_{1}-\sum_{i=2}^{n} M_{i}\right) \times x \\
& \text { or. } P=M \times \Gamma
\end{aligned}
$$

and we can solve for Vs: (Equation 7)

$$
F=\left(M^{T} \times M^{-1} \times\left(M^{T} \times F\right) .\right.
$$

Thereafter, we can compute the location of a feature on which the stylus tip is located by using Equation 1 or 2.

\section{Patient Registration}

The patient registration step is necessary to obtain a common frame of reference between the transmitter and (CT or MR) imaging spaces. In this step we compute the transformation matrices relating the various frames of reference that are involved. By locating features in the imaging study with the stylus, (either anatomical landmarks or temporary fiducial markers), in transmitter space, we have several points for which we know coordinates in both reference frames. Given n points (typically four) for which we have coordinates in CT space $\left(\mathrm{Pc}_{\mathrm{i}}\right)$ and in transmitter space $\left(\mathrm{Pt}_{\mathrm{j}}\right)$, because they are related by a single transformation such as in Equation 2, we can write an expression with augmented matrices: (Equation 8)

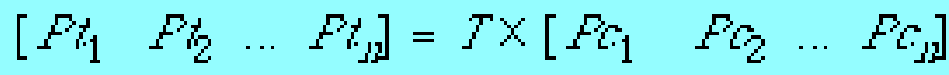

$$
\begin{aligned}
& \text { or. } F \text { ta }=T \times P G
\end{aligned}
$$

From Equation 8 we can compute the transformation matrix, T: (Equation 9)

$$
T=\left(F, a \times F c a^{T}\right) \times\left(F c a \times F c a^{T}\right)^{-1}
$$

The transformation matrix is used to map from image space to transmitter space. Its inverse, $\mathrm{T}^{-1}$, can be used to move in the other direction. One of the advantages of using affine matrices is that several of them can be used in series to perform complicated transformations that involve several frames of reference. For example, a point appearing in an ultrasound image (that is, in image space) (Pp) can be expressed in $\mathrm{CT}$ space $(\mathrm{Pc})$ by using the expression: (Equation 10)

$$
F c=v T_{p} \times r T_{j} \times r T_{p} \times F F
$$

where the sub- and superscript values applied to $\mathrm{T}$ correspond to the image, receiver, transmitter, and CT spaces.

\section{Free-Hand Ultrasound Image Registration}

We are ultimately seeking to assign a 3-D coordinate in some frame of reference to each pixel in an 
ultrasound image. By rigidly coupling our 3-D tracker to the imaging scanhead, we can keep track of its position in space. Although the scanhead and receiver are in fixed positions with respect to each other, we do not know exactly how they are related. We need to compute the offset vector between the receiver origin and the image origin, as well as a set of rotation angles for the image coordinate system with respect to that of the receiver. The process is similar to the stylus tip calibration except that there are now six degrees of freedom to fix instead of three. This problem has been addressed by other groups $[4,10]$ by using various approaches. The method described by Prager and coworkers[10] requires a complicated calibration apparatus and procedure, whereas that described by Detmer and associates[4] is much simpler. Our calibration procedure was originally based on the method described by Detmer and associates, but we derived a direct method of computation that does not require iteration. The main difference between our method and Detmer and associates' is that we do not enforce scaling and orthogonality constraints in our computations. In numerical tests, we have found that both methods produced nearly identical results, and thus, we opted for the simpler one.

Our calibration procedure is based on acquiring multiple images typically between 50 and 100 of a single point in a tank while holding the scanhead in as many different positions as possible to exercise all of the degrees of freedom. The calibration point consists of the intersection of two thin nylon wires positioned in a water tank (32 cm wide x $64 \mathrm{~cm}$ long and $46 \mathrm{~cm}$ deep) and that is clearly visible (Fig. 1).

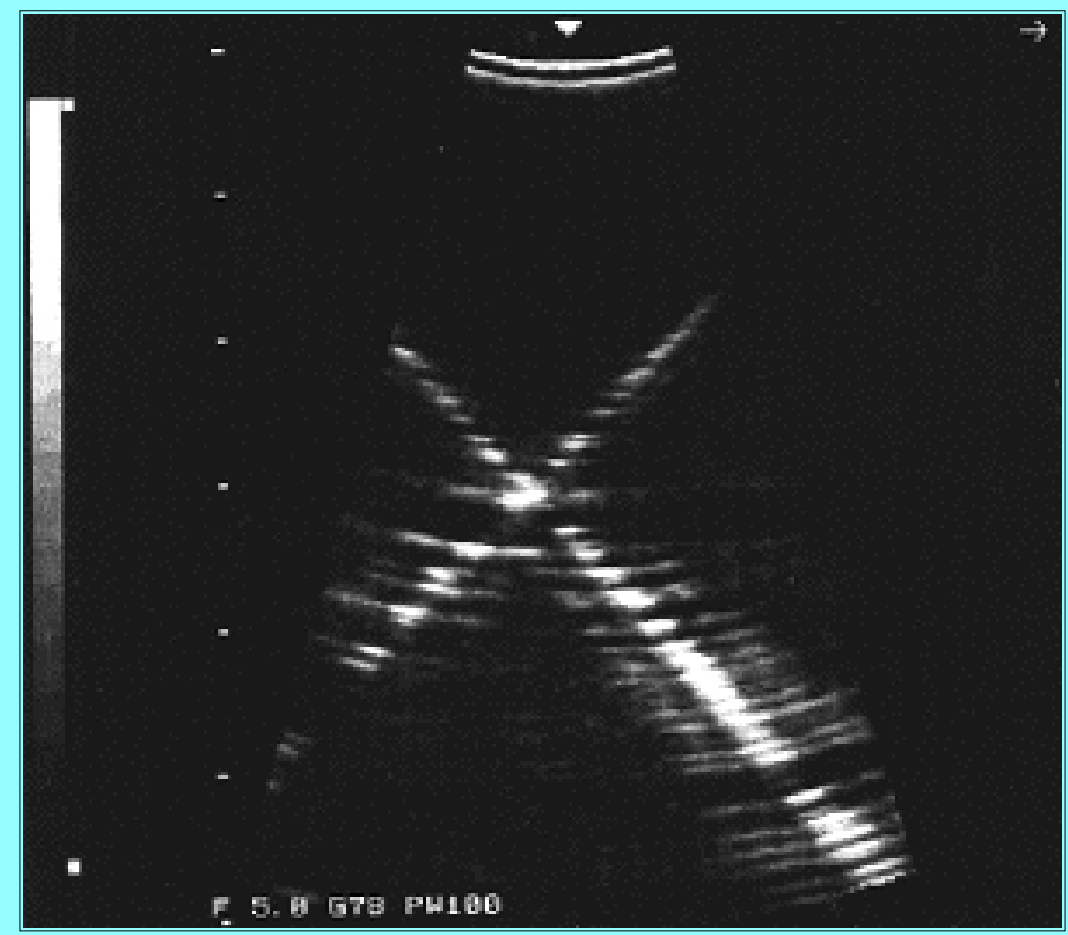

Fig. 1. Ultrasound image depicting the apperance of calibration point.

The endpoints of the two wires are recorded using the stylus in transmitter space, and the intersection is computed. This method of determining the intersection results in a smaller error than if we applied the stylus directly at the intersection, because of the effective division of the error that takes place (this can be thought of as a reverse-lever-arm effect). Because the intersection point's coordinates are known in both transmitter and image spaces, we are able to compute the six degrees of freedom in which a point in a picture is related to the receiver coordinate system. Assuming that the images are scaled properly, we begin with a simplified version of Equation 10, relating the location of a point in a picture $(\mathrm{Pp})$ and that point's location in transmitter space $(\mathrm{Pt})$, with the index $i$ that represents the image number: (Equation 11) 


$$
P t=r T_{J H} \times H T_{f} \times F P_{j}
$$

Here one should note that the coordinates of the calibration point (the wire intersection) in image space $\left(\mathrm{Pp}_{\mathrm{i}}\right)$ will vary while its position in transmitter space $(\mathrm{Pt})$ is fixed. Similarly, the transformation in which receiver and transmitter spaces are related $\left({ }^{(} T_{r}\right)$ will change with every image, while the relationship between image and receiver spaces $\left({ }^{(} \mathrm{T}_{\mathrm{p}}\right)$ is fixed but unknown. Another detail to keep in mind is that we use 4-tuples to designate 3-D coordinates resulting in vectors of the form $[\mathrm{x} \mathrm{y} \mathrm{z} 1]^{\mathrm{T}}$. For points in an image, only two coordinates are available (that is, pixel row and column). We chose arbitrarily to define the $\mathrm{z}$ axis as perpendicular to the image plane and set all $\mathrm{z}$ values for image coordinates at 0 . As a result, all image coordinates are represented by a four-tuple of the form $\left[\begin{array}{lll}\mathrm{x} & \mathrm{y} & 1\end{array}\right]^{\mathrm{T}}$. Because of the consistently 0 -valued $\mathrm{z}$ component, it is not possible to use an approach similar to that developed for patient registration (Equation 9) because the last term becomes singular. Instead, we must expand matrix Equation 11 isolate all of the unknown values in a vector, and then rewrite the expression in a form that can be solved by matrix inversion. For each image we can write the following equation that relates a point in the image (Pp) and its location in the transmitter $(\mathrm{Pt})$ frame; the index i corresponds to images: (Equation 12)

$$
\begin{aligned}
& P t=r T_{j H} \times r T_{p} \times P P_{j} \\
& \text { or: } \quad T_{H J}-1 \times F_{t}=T_{f} \times P_{P_{j}} \\
& \text { or. } \quad F_{t}=F_{f} \times F_{p}
\end{aligned}
$$

The left side collapses to a vector $\left(\mathrm{Pt}_{\mathrm{i}}\right)$ for each image, whereas the right side is the product of $\mathrm{a}_{4} \mathrm{X} 4$ matrix by $\mathrm{a}_{4 \mathrm{X} 1}$ vector whose components we expand: (Equation 13)

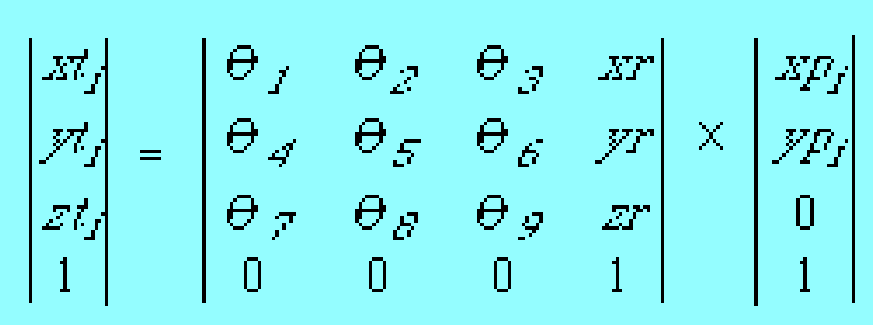

In this equation, the unknown values we seek are $\theta_{1}, \theta_{2}, \theta_{4}, \ominus_{5}, \ominus_{7}, \theta_{8}$ xr, yr, and zr. The remaining variables, $\theta_{3}, \theta_{6}$, and $\theta_{9}$, are immaterial because they multiply the $\mathrm{z}$ coordinate, which is always 0 . We can rewrite this equation in the following form, which separates the unknowns into one vector and arranges the known values into a matrix: (Equation 14) 


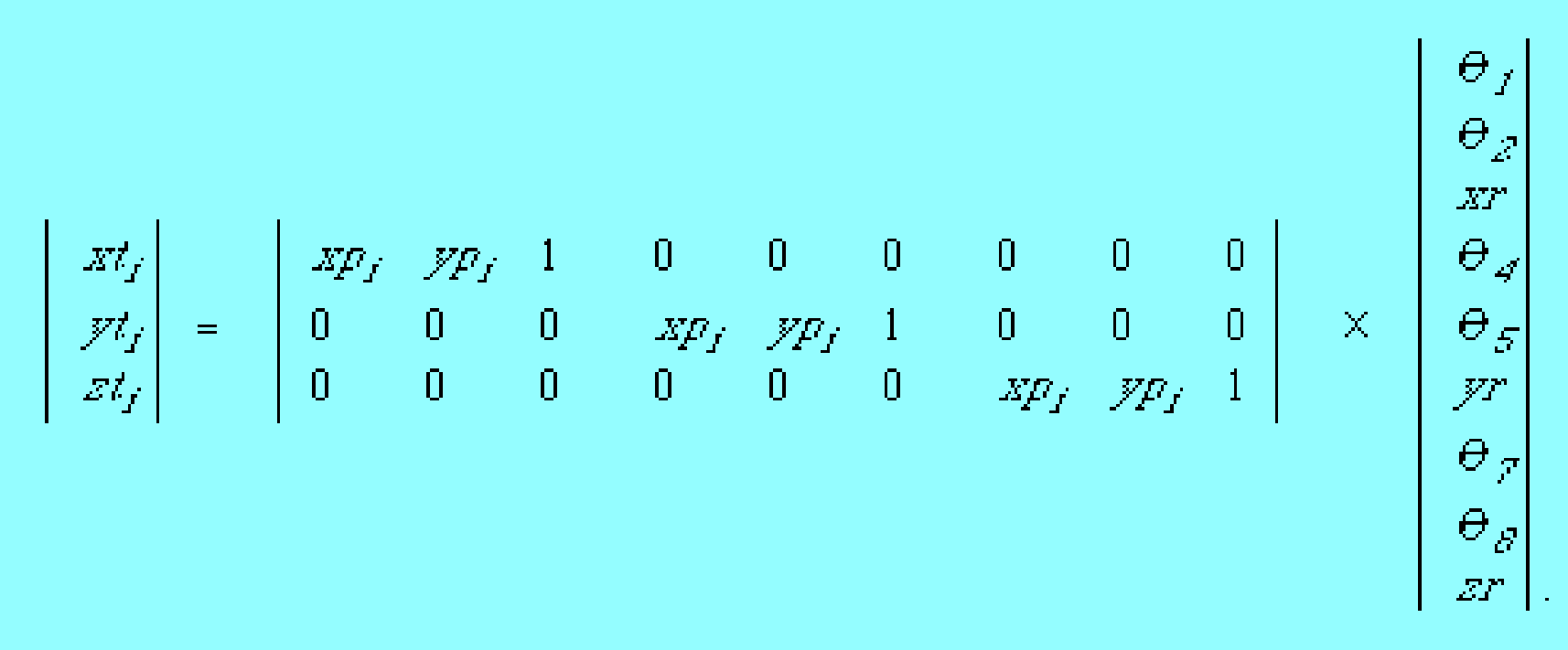

With several images showing the same point, we can write an augmented matrix: (Equation 15)

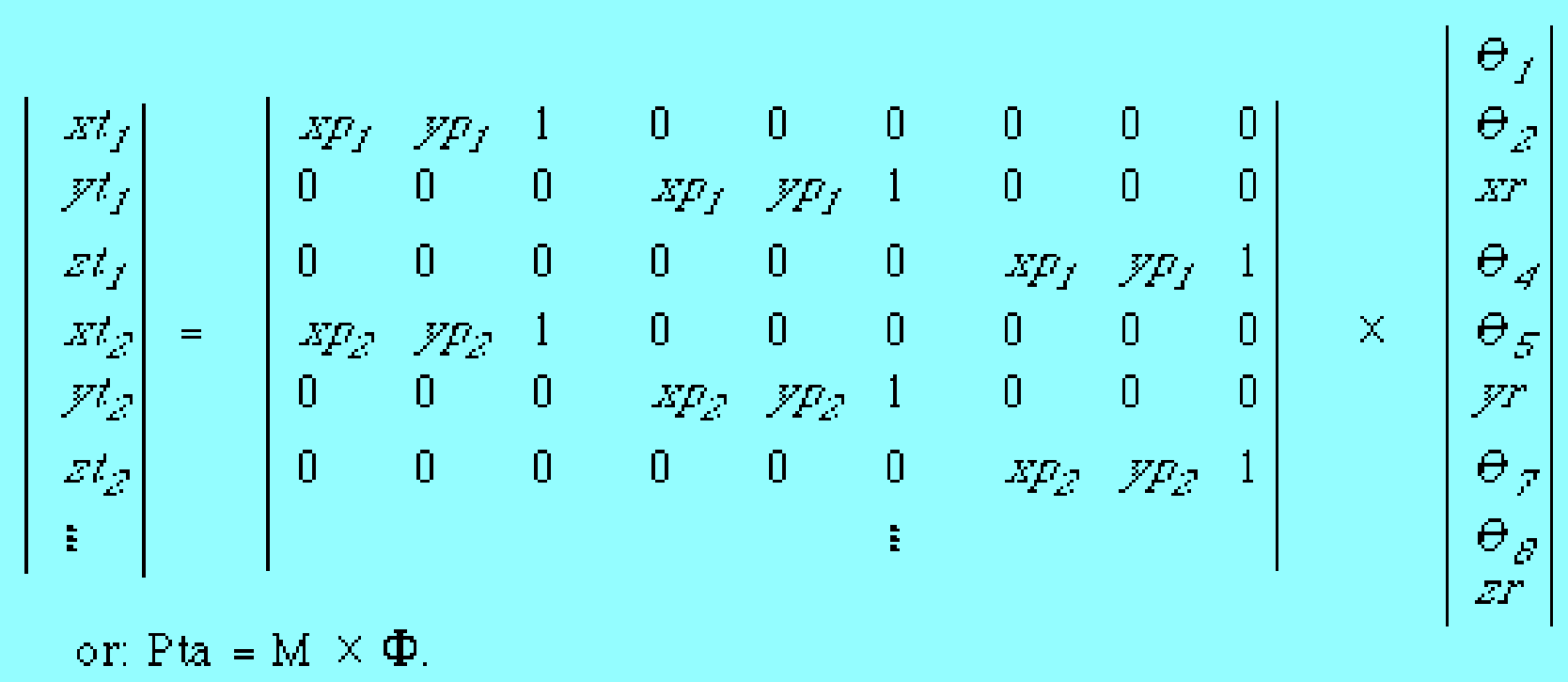

We can then solve all of the unknowns in $\mathrm{O}$ by using the least-square estimate inversion of $\mathrm{M}$ : (Equation 16)

$$
\Phi=\left(A^{T} \times A^{-1} A^{T} \times P \hbar\right.
$$

Notice that in this formulation of the calibration process we are not restricted to using a single calibration point. Any number of points could be used, in fact, as is apparent in Equation 14. We use a single point that consists of the intersection of two wires in a tank, which is much more readily identified in an ultrasound image (Fig. 1). By using a single point the values in vector Pta are repeated.

\section{RESULTS}

The accuracy of our setup is dependent on the error that results from each step of the process. The FOB position tracking system used in our study has a high reported overall accuracy of $1.78 \mathrm{~mm}$ for translations and $0.5^{\circ}$ for rotations. These reported figures are much better than those by Birkfellner, et al.[2] In our experiments we have observed that the FOB is adversely affected by the presence of large ferromagnetic objects. Nonferromagnetic metals such as aluminum and stainless steel, which are 
predominantly used in the OR, seem to have much less effect on the accuracy of the FOB, as was verified by Birkfellner, et al. Any inaccuracies inherent to the tracking device and amplified by the clinical environment would be apparent in the stylus-calibration and patient-registration steps of our process.

\section{Stylus Calibration Accuracy}

Because it is not physically possible to locate the origin of the transmitter coordinate system, it is also not possible to measure the location of any object in its frame of reference by using, for example, rulers. For this reason, we can only gauge the repeatability and the relative accuracy of our ability to locate the stylus tip. To estimate the repeatability of our stylus calibration procedure, we have performed a simple experiment in which we used the calibrated value of the stylus tip position (the offset vector) and recorded position/orientation data from the FOB to compute the resulting tip location, following a calibration procedure based on 10 points. The resulting computed points in transmitter space will differ from the actual calibration point, due to errors. From these data we obtained a root mean square (RMS) error by computing the standard deviation $(\mathrm{SD})$ in the $(\mathrm{x}, \mathrm{y}, \mathrm{z})$ component values of the point coordinates and then computing the square root of the sum of squares of the SDs in the three dimensions: (Equation 17)

$$
F_{Y M Y}=\sqrt{\sigma_{r}^{2}+\sigma_{Y y}^{2}+\sigma_{z}^{2}}
$$

We found the RMS error (Erms) to be $0.61 \mathrm{~mm}$ in 30 experiments.

In a second experiment we placed the stylus tip on markings on the test tank along three of its edges that corresponded roughly to the $\mathrm{x}, \mathrm{y}$, and $\mathrm{z}$ directions of the transmitter space. The markings were spaced 1 inch $(25.4 \mathrm{~mm})$ apart, and we measured 13 markings in each direction. We computed the distances between consecutive markings and subtracted from those values the nominal separation of $25.4 \mathrm{~mm}$. We computed the average and SD of the absolute values of the resulting error and found them to be $1.36 \pm$ $1.67 \mathrm{~mm}$ for a total of 39 points.

\section{Patient Registration}

We tested the patient registration procedure by using a phantom consisting of a specially built Plexiglas cylinder (14-cm diameter by $16-\mathrm{cm}$ height) with 20 nylon screws evenly spaced around it. We acquired a CT scan of the phantom and obtained the screwhead locations based on the pixel size and slice spacing. The scan consisted of 132 slices at $1.25-\mathrm{mm}$ spacing with a pixel size of $0.4 \mathrm{~mm}$ in $\mathrm{x}$ and $\mathrm{y}$. The phantom was intended to be approximately the size of a human head. After placing it in the same head clamp that is used with patients, we performed the measurements in the OR. Following the normal procedure for calibrating the stylus, we recorded the position of the 20 screwheads on the exterior of the phantom by pointing at them with the stylus. To gauge the accuracy of the registration computation, we computed the transformation matrix relating the CT and transmitter spaces as outlined in Equations 8 through 10. We selected four out of the 20 screws as "fiducials" to compute the transformation matrix. Using the transformation matrix, we then computed the coordinates for the screwheads in CT space. By comparing the actual locations of the screwheads in CT space with the computed values, we found an error of $0.86 \pm$ $0.61 \mathrm{~mm}$ (for 20 screws), which includes the stylus tip uncertainty.

\section{Free-Hand Ultrasound Image Registration}


The ultrasound image registration step is intended to provide the location in 3-D space of any feature appearing on an ultrasound image. To test how accurately we can compute a feature's location in transmitter space, we performed two experiments, again trying to circumvent the impossibility of obtaining an accurate absolute and independent measurement of position in transmitter space.

The first experiment follows our normal calibration procedure that is based on obtaining 70 to 100 images of the intersection of two nylon wires in a large tank (Fig. 2).

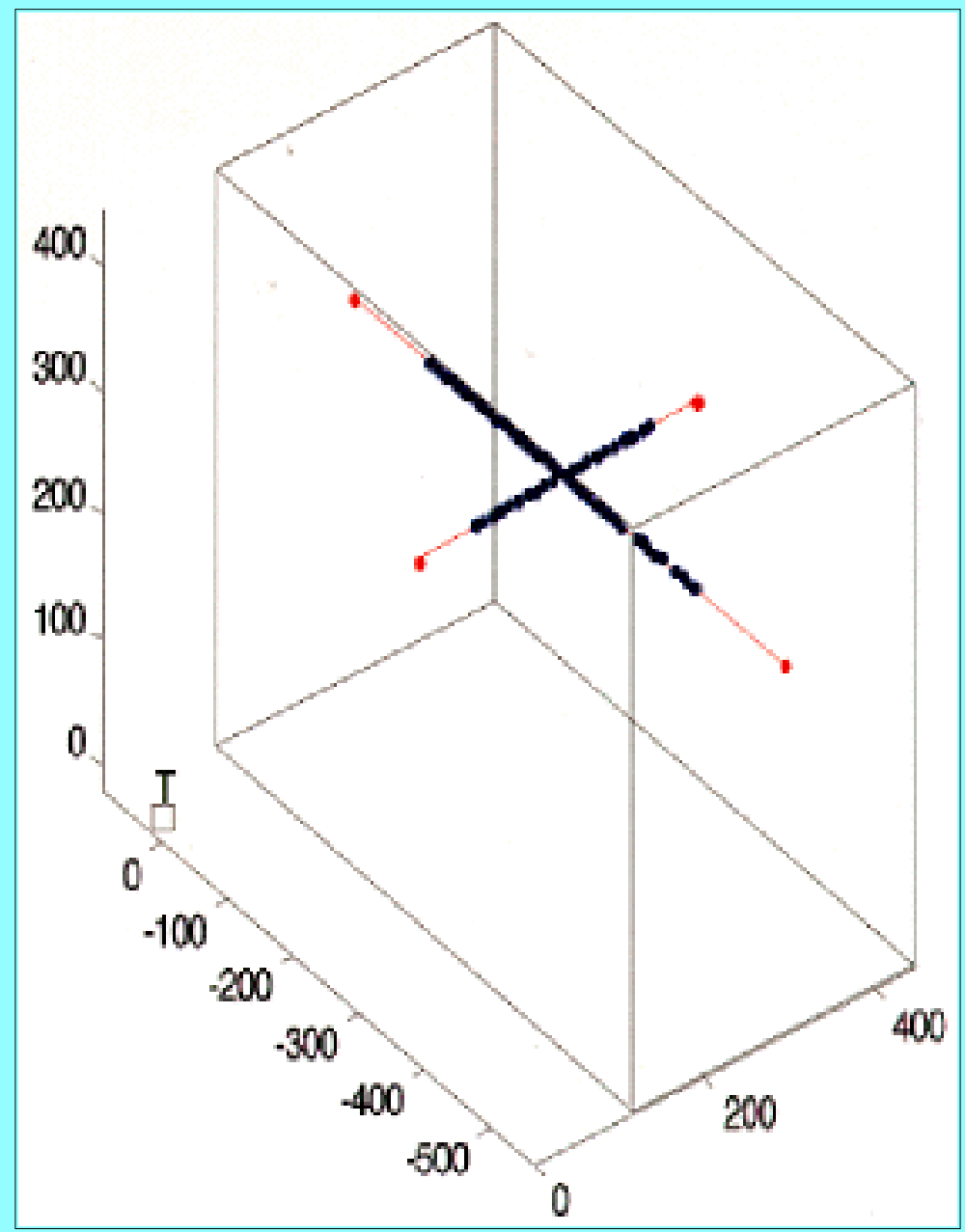

Fig. 2. Schematic representation of the ultrasound image calibration tank. The transmitter is located at the origin $(\mathrm{T})$. Black lines indicate the tank edges; red the nylon wires; and blue, the beads.

The location of the calibration point is obtained by measuring the location of the endpoints of the wires in the tank and computing their intersection. Using the transformation matrix obtained from the calibration process, which is outlined in Equations 11 to 16, we then computed the location of the calibration point in transmitter space. From the resulting set of points, we compute an "average point," which is located at the centroid of the cloud of points. The average point is subtracted from all the computed points to provide a set of error vectors $\mathrm{DP}_{\mathrm{i}}$.

The RMS error is then computed from: (Equation 18) 


$$
\text { HWMS }=\sqrt{\frac{1}{M} \sum_{j=1}^{H}\left(\Delta \mathrm{P}_{i}^{T} \times \Delta P_{j}\right)} .
$$

In this experiment, we used 93 different images and obtained a RMS error of $2.12 \mathrm{~mm}$.

Another, and more revealing test is to calculate the transmitter space position of features that were not used in the image calibration based on their ultrasound images. We used 60 beads strung on the wires at fixed positions and at regular intervals (see Fig. 2: two of the beads came loose and were not used in the experiments). We acquired images of all the beads with the scanhead as well as of their actual locations with the stylus tip. The position of the beads and their corresponding computed positions are shown in Fig. 3.

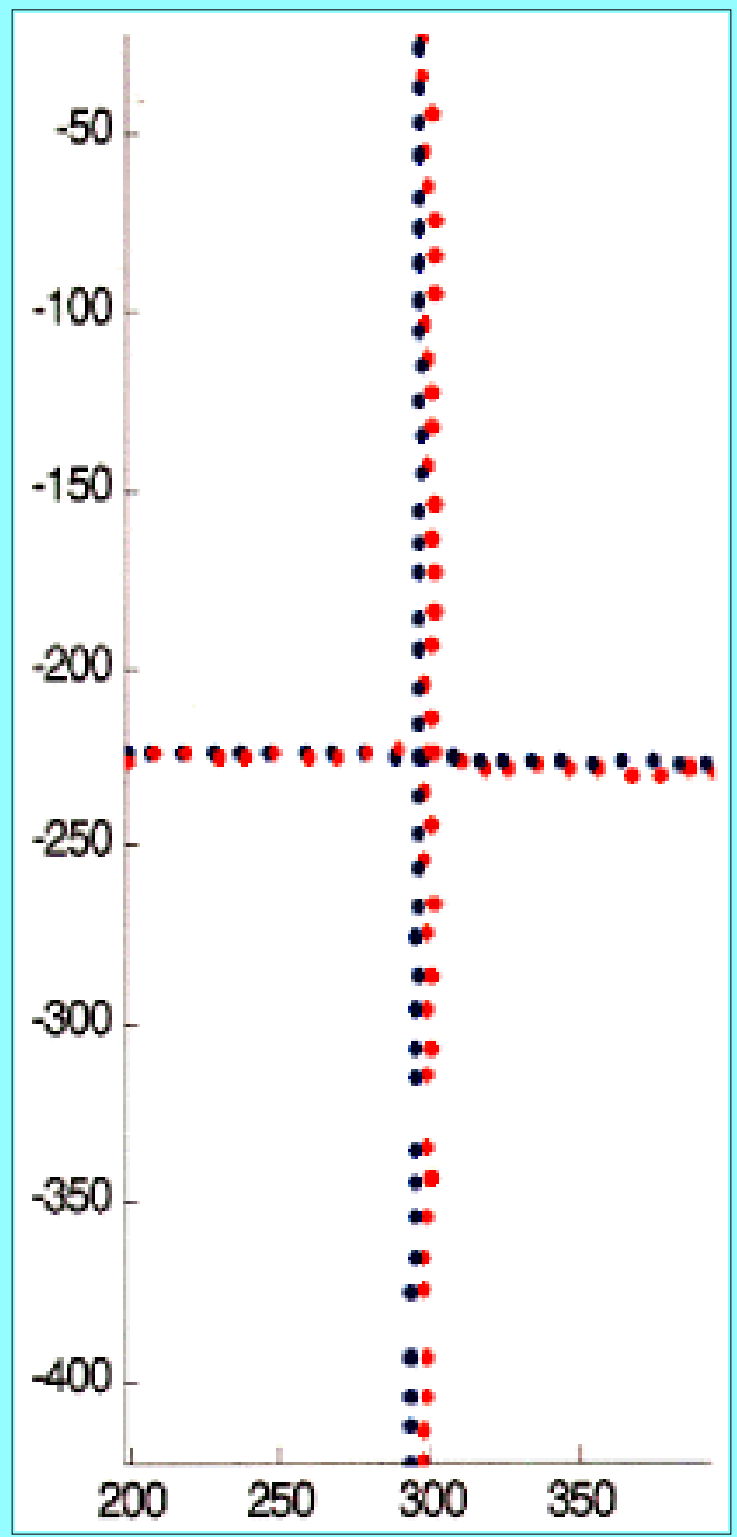

Fig. 3. Bead locations seen from above the tank showing measurement disagreement between. Blue dots indicate the stylus (measured) and red dots the ultrasound (calculated). 
We were also able to measure the bead positions with respect to the intersection (calibration) point with a ruler along the wires. With these data we were able to compare the consistency of the two procedures: stylus pointing and 3-D ultrasound. In this test the measured 3-D location of each bead, as established either by the stylus or ruler, is subtracted from its respective position that was obtained via the 3-D ultrasound to arrive at the error vector $\mathrm{DP}_{\mathrm{i}}$. We found the average magnitude of the error vectors to be $2.96 \pm 1.85 \mathrm{~mm}$ (based on 58 beads).

\section{DISCUSSION}

This series of experiments was conducted to discern the overall accuracy of our 3-D ultrasound system to help determine the appropriateness of its use in updating imaging data for the purpose of neuronavigation. The sources or possible error are many and, assuming that the methodology and computations are correct, can be summarized as follows: 1) errors due to the 3-D tracking system: these include overall accuracy and repeatability under normal conditions (that is, no metals present in the surroundings) as well as error introduced by the presence of the usual objects in the OR: (head clamp, OR table, and other instruments); 2) errors due to the ultrasonography apparatus: these consist of possible errors in scaling in the ultrasound images and possible internal distortions in the system's procedure for translating echo data into pixel intensities; 3) ultrasound image thickness; and 4) manual errors: these consist of slightly missing the target when pointing the stylus to a point, a bead, a fiducial marker, or an anatomical feature.

The first type of error listed is the most important because every step of our procedure depends on the tracker's accuracy. In their study, Birkfellner, et al.,[2] undertook a systematic investigation of two types of tracking devices (AC compared with DC) and their results have characterized the overall expected accuracy of our system, which is a DC-pulsed magnetic tracker to be $6.4 \pm 2.5 \mathrm{~mm}$ for distance and $4.9^{\circ}$ $\pm 2.0^{\circ}$ for rotation in an OR environment. These values are less accurate than those observed in our experiments, although the methodologies are quite different. Our results, based on the stylus tip calibration, indicate an overall accuracy of $1.36 \pm 1.67 \mathrm{~mm}$, or approximately one-third of their reported error. Their experiments were based on carefully built six degrees of freedom nonmetallic racks, whereas ours were free-hand either in the laboratory or in the OR. It should be noted that the calibration procedure for the stylus tip would remove any systematic linear offset in either distance or rotation measurements that may exist in the overall operation of the 3-D tracker, because these would effectively be subtracted. The effect of distortions (nonlinear scaling errors or warping) is not as easily accounted for in the calibration, and it is expected that it will be reflected in the overall accuracy. The same correction will result in the calibration of the ultrasound image to receiver frames transformation. Patient registration, which is based on locating identical points in both the MR imaging or CT spaces as well as in the transmitter space, depends on properly identifying these points in both cases. Error will result if the points are improperly identified on the MR imaging. This can result from relatively large slice spacing or simply misidentification of features.

Errors due to distortions in the recorded ultrasound images would only affect the last part of our procedure, which is the registration of ultrasound images in transmitter space. Internally, the ultrasound imaging unit transforms a series of A-scan echo waveforms into intensity images. This process is based on the propagation time of an acoustic wave in tissue and on the knowledge of the angle of the A-scan with respect to the scanhead. Both of these parameters can be in error, resulting in two types of error; scaling error and distortion. Neither of these would be corrected by our registration procedure, and therefore we depend on proper calibration of the imaging unit by using phantoms of known dimensions. 
Although one conceives of an image as being perfectly flat and dimensionless in the direction perpendicular to its plane, ultrasound images reflect acoustic disturbances over a finite thickness of tissue. This thickness tends to increase with the distance from the scanhead. We have conducted a simple experiment in which we translated the scanhead over a ruler while observing over what distance a fixed feature in the tanks was visible. With this method we obtained on image thickness of $4 \mathrm{~mm}, 4 \mathrm{~mm}$, and 8 $\mathrm{mm}$ at distances from the scanhead of $19 \mathrm{~mm}, 37 \mathrm{~mm}$, and $44 \mathrm{~mm}$, respectively. This result is mitigated by other factors. For example, highly echogenic features will tend to show up more readily and be visible at greater distances from the image ideal plane. The finite thickness of the image slice can introduce error in our image-registration procedure because features that appear in an image are assumed to be intersecting the ideal plane of the picture when, in fact, they may be located some distance above or below it, violating the assumption that their $\mathrm{z}$ coordinate is always 0 . To reduce the effect of image thickness in our calibration procedure as much as possible, we swept the scanhead across the reference point to estimate the middle of the range in which it was visible, attempting to acquire the images in that position. This is very much a user-dependent process, but presumably thickness error operates both above and below the plane of the images and will tend to cancel with a large number of images.

Manual error when pointing to a feature will also contribute to errors in our procedure. We tried to reduce this in the tank-calibration procedure by pointing to hard features on the tank's wall whenever possible. This was the case when we measured the location of the endpoints of the wire segments rather than their intersection directly. This type of error is more difficult to avoid on targets that can move, such as a feature on the skin or a bead on a string. The possibility of moving the target object will introduce error. Misidentification of features will result in the same type of errors.

We found that we were able to locate in a fixed reference frame features that appeared in a free-hand ultrasound image with an accuracy of $2.96 \pm 1.85 \mathrm{~mm}$ based on measurements made in a water tank. We have also found that in using a rigidly coupled stylus with our 3-D tracker, we can locate a point in transmitter space with an accuracy of $1.36 \pm 1.67 \mathrm{~mm}$. Both these values indicate that we obtained much better performance from our system than could be expected from the results presented by Birkfellner. We hypothesize that the calibration procedures we use improve the system's operation insofar as they can eliminate consistent linear bias in both distance and rotation measurements.

Preliminary experiments in patients, which have been approved by our internal review board, demonstrated that 3-D ultrasound imaging is practical. Fig. 4 provides an illustration of the technique in which the plane defined by the ultrasound image (Fig. 4 left) has been reconstructed from MR imaging data by using an oblique plane intersection in which ANALYZE (Biomedical Imaging Resources, Mayo Foundation, Rochester, MN) is used. 

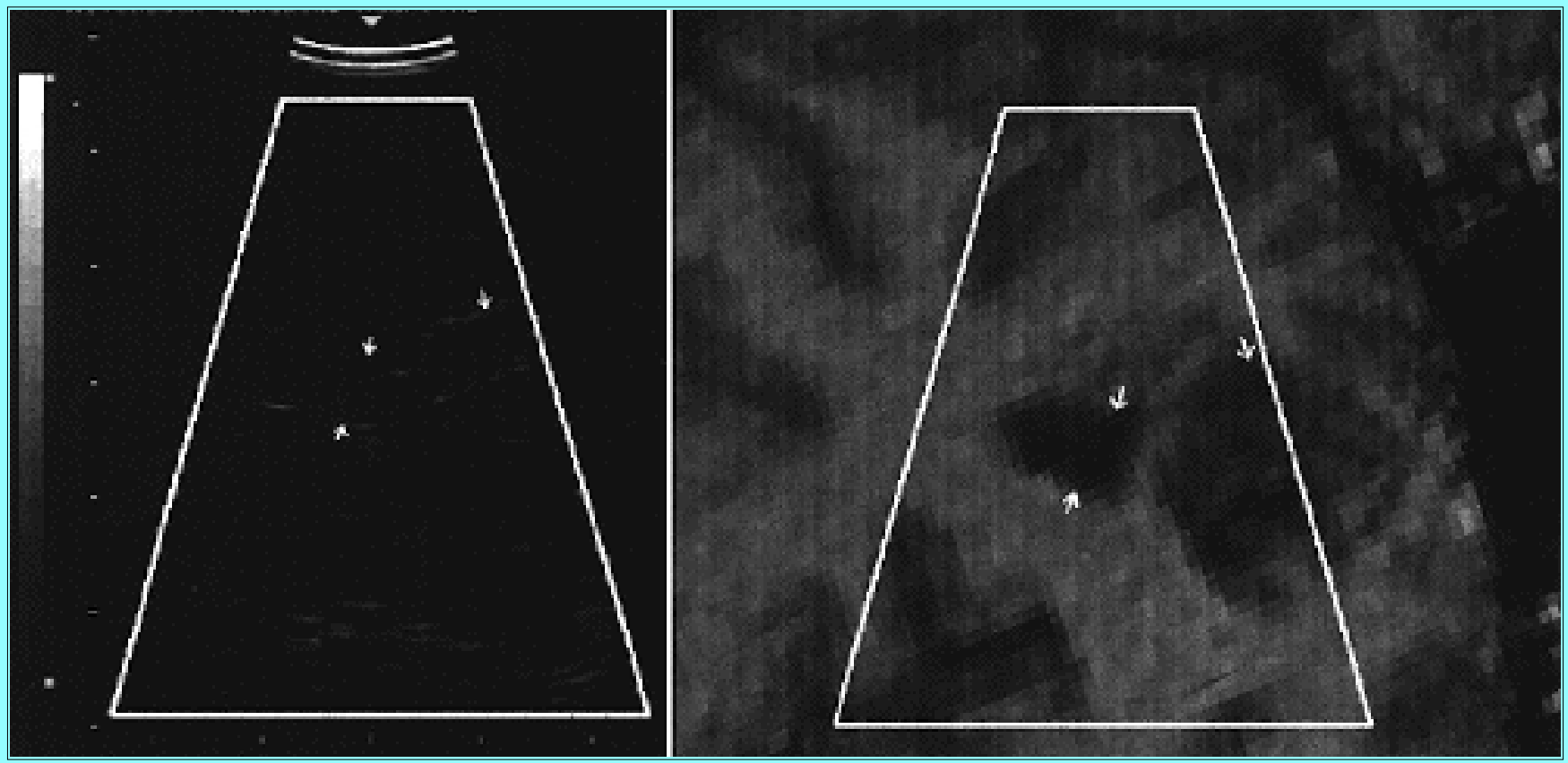

Fig. 4. Recomputation of the plane of intersection corresponding to the ultrasound image (left) in the MR imaging study (right). The outline indicates the exact location of the image. Two features have been identified and are shown between the arrows in the center and, below, the single arrow to the right on both images.

The outline of the ultrasound image is visible in both left and right images, and arrows indicate features that have been identified on both images. Given our previous findings of typical brain displacement on the order of $1 \mathrm{~cm}$ during surgery,[12] we believe that the accuracy of our system would warrant using it in updating imaging information on which neuronavigation is based.

\section{References}

1. Ault T, Siegel MW: Frameless patient registration using ultrasonic imaging: a preliminary study. J Image Guided Surg 1:94-102, 1995

2. Birkfellner W, Watzinger F, Wanschitz F, et al: Systematic distortions in magnetic position digitizers. Med Phys 25:2242-2248, 1998

3. Comeau RM, Fenster A, Peters TM: Intraoperative US in interactive image-guided neurosurgery. Radiographics 18:1019-1027, 1998

4. Detmer PR, Bashein G, Hodges T, et al: 3D ultrasonic image feature localization based on magnetic scanhead tracking: in vitro calibration and validation. Ultrasound Med Biol 20:923-936, 1994

5. Dorward NL, Alberti O, Velany B, et al: Postimaging brain distortion: magnitude, correlates, and impact on neuronavigation. J Neurosurg 88:656-662, 1998

6. Gänser KA, Dickhaus H, Staubert A, et al: [Quantification of brain shift effects in MRI images.] Biomedizin Techn (Suppl):247-248, 1997 (Ger)

7. Hill DLG, Maurer CR Jr, Maciunas RJ, et al: Measurement of brain surface deformation under a craniotomy. Neurosurgery 43:514-528, 1998 
8. Leotta DF, Detmer PR, Martin RW, et al: Performance of a miniature magnetic position sensor for three-dimensional ultrasound imaging. Ultrasound Med Biol 23:597-609, 1997

9. Paulsen KD, Miga MI, Kennedy FE, et al: A computational model for tracking subsurface tissue deformation during stereotactic neurosurgery. IEEE Trans Biomed Eng 46:213-225, 1999

10. Prager RW, Rohling RN, Gee AH, et al: Automatic calibration for 3-D free-hand ultrasound. CUED/F-INFENG/TR 303. Cambridge University Engineering Department. Work in Progress Report, Sept 1997. http://www.eng.cam.ac.uk/reports/abstracts/prager_tr303.html

11. Roberts DW: Frameless stereotaxy. Concepts Neurosurg 7:78-84, 1995

12. Roberts DW, Hartov A, Kennedy FE, et al: Intraoperative brain shift and deformation: a quantitative analysis of cortical displacements in 28 cases. Neurosurgery 43:749-760, 1998

13. Roberts DW, Strohbehn JW, Friets EM, et al: The stereotactic operating microscope: accuracy refinement and clinical experience. Acta Neurochir Suppl 46:112-114, 1989

14. Wirtz CR, Bonsanto MM, Knauth M, et al: Intraoperative magnetic resonance imaging to update interactive navigation in neurosurgery: method and preliminary experience. Comput Aided Surg 2:172-179, 1997

Manuscript received January 20, 1999.

Accepted in final form February 2, 1999.

This work was supported by Grant No.R01-NS33900-02 fromm the National Institute of Health.

Address reprint requests to: Alexander Hartov, Ph.D., Thayer School of Engineering, Dartmouth College, HB 8000, Hanover, New Hampshire. email: alex.hartov@dartmouth.edu.

\section{COMMENTARY ON ARTICLE}

The following references are suggested by the editors for those interested in pursuing this topic further.

\section{References}

1. Barry CD, Allott CP, John NW, et al: Three dimensional freehand ultrasound: image reconstruction and volume analysis. Ultrasound Med Biol 23:1209-1224, 1997

2. Leggett ME, Leotta DF, Bolson EL, et al: System for quantitative three dimensional echocardiography of the left ventricle based on a magnetic-field position and orientation sensing system. IDDD Trans Biomed Eng 45:494-504, 1998

3. Leotta DF, Detmer PR, Martin RW, et: Performance of a miniature magnetic position sensor for three dimensional ultrasound imaging. Ultrasound Med Biol 23:597-609, 1997

4. Prager RW. Rohling RN, Gee AH, et al: Rapid calibration for 3-D freehand ultrasound. Ultrasound Med Biol 24:855-869, 1998

5. Rohling RN, Gee AH, Berman L: Automatic registration of 3-D ultrasound images. Ultrasound Med 
Biol 24:841-854, 1998 